\title{
Design of the Pulse Oximetry Measurement Circuit and Its Sensing System Based On CMOS
}

\author{
Yang Wang ${ }^{1}$, Liying Chen ${ }^{2}$ \\ ${ }^{1}$ (Tianjin Poly-technic University, School of Electronic and Information Engineering, 300387, China) \\ ${ }_{2}^{2}$ (Tianjin Poly-technic University, School of Electronic and Information Engineering, 300387, China)
}

\begin{abstract}
The pulse oximetry circuit and its sensing system is designed based on the standard CMOS technology of 0.18um. The reflection oxygen sensor is used to collect the pulse oximeter signal of human body, then the collected physiological signals are processed by the data processing circuit The data processing circuit is composed of two parts: the amplifying circuit and the band-pass filter circuit, and the pulse oximeter data processed by the data processing circuit is written into the tag through the SPI communication The RFID reader read the data in the RFID tag through wireless communication, and display the data. The experimental results show that the maximum error is $\pm 1 \%$. The maximum error of the pulse is $\pm 1.9 \%$. The stability and feasibility of pulse blood oxygen sensing system is demonstrated in this paper and it will have a good application prospect in the direction of wearable medical wisdom research.
\end{abstract}

Keywords: measurement circuit, pulse oximetry, wireless sensor networks, reflection type, active RFID tag

\section{Introduction}

With the aging of the society is becoming more and more serious, the cost of medical care for the elderly and the proportion of medical resources is increasing. In order to alleviate the pressure on the health care system, the traditional medical center model needs to be transformed into a family centered model. Wearable medical device is a device that integrates signal acquisition, processing and data transmission. The physiological parameters such as pulse, oxygen saturation and temperature were collected, processed and analyzed by the biomedical sensors on the human body. The data can be displayed timely feedback to the user in the device, and can also be used for real-time monitoring of users. It does effectively carry out the prevention and treatment of disease.

At present, the most mature technology in the detection of blood oxygen saturation [1] in the human body is the transmission detection structure. However, the reflection type instrument has been paid more and more attention, because of the limitation of the current type of testing instruments in the acquisition of the human body. The light source and the light detector of the reflective pulse oxygen sensor is placed on the same side of the detection part. The detector receives the light reflected back to human tissue, and convert optical signals into electrical signal as the output of the sensor. The reflective optical detection mode is less limited by the detection position, so that the detection position is more flexible. The measurement can be achieved in despite of the limit of position.

With the development of medical sensor technology, the transmission type detection method of pulse oximetry has been gradually replaced by reflective detection mode. It has become the main measurement method for detecting pulse oximetry, and received great attention and research in the field of medicine. In this paper, the reflective probe is used to collect the signal, and the measurement circuit is designed with UMC 180nm CMOS standard technology. After the amplification and filtering of the collected signal, the signal processed by the circuit will be transmitted further to other modules.

\section{Related Works}

The components of the absorption of light in the blood mainly include oxygenated hemoglobin ( $\mathrm{HbO} 2)$ and reduced hemoglobin $(\mathrm{Hb})$. Blood oxygen saturation is defined as the percentage of $\mathrm{HbO} 2$ in the blood, which is the sum of the concentration of $\mathrm{HbO} 2$ and the concentration of $\mathrm{Hb}$ in the blood. The concentration of $\mathrm{HbO} 2$ is an important physiological parameter for the metabolism of human tissues. The definition of blood oxygen saturation:

$$
\mathrm{SpO}_{2}=\frac{C_{\mathrm{HbO}_{2}}}{C_{\mathrm{HbO}_{2}}+C_{\mathrm{Hb}}} \times 100 \%
$$

In the formula, $\mathrm{Spo}_{2}$ represents the value of oxygen saturation, $\mathrm{C}_{\mathrm{Hb} \text { o2 }}$ represents the concentration of $\mathrm{HbO} 2$ in the blood, and $\mathrm{C}_{\mathrm{Hb}}$ indicates the concentration of $\mathrm{Hb}$ in the blood. Bill Lambert's law states that light has certain attenuation characteristics in non-homogeneous scattering medium, the absorption degree of the light in human issues has certain difference in different medium. The measurement of oxygen saturation $[2,3]$ is based on 
Lambert Bill's law as the theoretical basis, the light passes through the medium of interference without considering the absorption of skin and muscle and other tissue to light condition. The blood $\mathrm{HbO} 2$ and $\mathrm{Hb}$ have different absorption rates of different wavelengths. Different wavelengths of light can emit the device as a light source. We can calculate the content of hemoglobin in blood components through the measurement of the attenuation degree after the light passing through the blood.

The measurement of the pulse and blood oxygen saturation is based on the principle that the light absorption quantity will change with the expansion or contraction of the artery, while the light absorption of other organizations are almost constant. The amount of light absorbed by the arterial blood changes when the arterial pulse. The amount of light absorbed by the skin, muscles, bones, venous blood, and non-pulsating arteries is constant. Heart contraction or expansion will affect the arterial volume, according to Lambert Bill's law. Change of the optical path will affect the absorbance, ripple component of artery is PPG (pulse wave) signal. According to the photon diffusion equation and time resolved spectroscopy technique, the formula of the reflected light intensity can be obtained:

$$
R(\rho, t)=\frac{\exp \left(-\mu_{a} c t\right) \exp \left(-\frac{\rho^{2}+\left[(1-g) \mu_{s}\right]^{-2}}{4 \pi D c}\right)}{(1-g) \mu_{s} \sqrt{(4 \pi D c)^{3} t^{5}}}
$$

When the time is long enough, the formula (2) is approximately equal to $\mu_{a} c, W$ that represents the change of the reflected light intensity, is directly proportional to the absorption coefficient $\mu_{s}$.

$$
W=\mu_{a} c
$$

According to the Lambert Bill law, when a beam of light intensity of $I_{0}$ is perpendicular to a sample with a concentration of $C$, the transmitted light intensity $I$ and $I_{0}$ exist.

$$
I=I_{0} e^{-\varepsilon(\lambda) C L}
$$

(4)

In the formula, $\varepsilon(\lambda)$ is the absorption coefficient of the sample molecule to the wavelength $\lambda ; L$ is the path of light. In order to carry out the transformation, the absorption coefficient $\mu_{a}$ is obtained.

$$
-\frac{1}{L} \ln \left(\frac{I}{I_{0}}\right)=\varepsilon C=\mu_{a}
$$

Assuming that $\varepsilon_{H b}$ and $C_{H b}$ are the absorption coefficient and concentration of $\mathrm{Hb}$ in blood, $\varepsilon_{\mathrm{HbO} 2}$ and $C_{\mathrm{HbO} 2}$ are respectively the absorption coefficient and concentration of $\mathrm{HbO} 2$ in blood. When the light irradiates on the human body with two kinds of wavelength of red and infrared light as light source, the absorption coefficient of $\mathrm{HbO} 2$ and $\mathrm{Hb}$ on the two wavelengths of light can type (5) respectively, only considering the effect of absorption of $\mathrm{HbO} 2$ in blood and $\mathrm{Hb}$.

$$
\begin{aligned}
& \mu_{a}{ }^{R}=\varepsilon_{\mathrm{Hb}}^{R} C_{\mathrm{Hb}}+\varepsilon_{\mathrm{Hbo}_{2}}^{R} C_{\mathrm{Hbo}_{2}} \\
& \mu_{a}^{I R}=\varepsilon_{\mathrm{Hb}}^{I R} C_{\mathrm{Hb}}+\varepsilon_{\mathrm{Hbo}_{2}}^{I R} C_{\mathrm{Hbo}_{2}}
\end{aligned}
$$

When light passes through human tissue and blood vessels, it can be divided into two parts: DC component and AC component. The change rate of light intensity in the tissue is $W=I_{A C} / I_{D C}$ binding (2), (6) and (7) According to the double beam method, the following formula will be derived.

The formula (9) is brought into (1),

$$
\begin{gathered}
\frac{W_{R}}{W_{I R}}=\frac{\varepsilon_{H b}^{R} C_{H b}+\varepsilon_{H b o_{2}}^{R} C_{H b o_{2}}}{\varepsilon_{H b}^{I R} C_{H b}+\varepsilon_{H b o_{2}}^{I R} C_{H b o_{2}}} \\
\frac{W_{R}}{W_{I R}}=\frac{I_{A C}^{R} / I_{D C}^{R}}{I_{A C}^{I R} / I_{D C}^{I R}}=A
\end{gathered}
$$




$$
\operatorname{spo}_{2}=\frac{\varepsilon_{H b}^{R}}{\varepsilon_{H b}^{R}-\varepsilon_{H b o_{2}}^{R}}-\frac{\varepsilon_{H b}^{I R}}{\varepsilon_{H b}^{R}-\varepsilon_{H b o_{2}}^{R}} \frac{W_{R}}{W_{I R}}
$$

In the formula, $\varepsilon_{\mathrm{Hb}}^{I R} \quad \varepsilon_{\mathrm{Hb}}^{R}$ and $\varepsilon_{\mathrm{Hbo}_{2}}^{R}$ are all constant, so we can get the formula of blood oxygen saturation:

$$
s p o_{2}=k_{1}-k_{2} A
$$

The reflective pulse oximetry can be calculated by the AC and DC information of the pulse wave signal.

\section{The Pulse Oximeter Measurement Circuit}

In this paper, the pulse oximetry sensor (DCM03) is used to detect the pulse oxygen saturation, which has the advantages of small volume, low power consumption and high precision. The key to measure the blood oxygen saturation is to get the pulse waveform. The pulse signal of human body is a physiological signal of low frequency and low amplitude, which is about $0.5 \mathrm{mV}$. In theory, the whole measurement system needs to be enlarged more than 200 times to achieve the requirement of ADC conversion voltage. The pulse frequency is concentrated in $0.5 \mathrm{~Hz} \sim 5 \mathrm{~Hz}$, and the maximum is less than $40 \mathrm{~Hz}$. In the process of signal acquisition, the movement of the body and mental stress will produce baseline drift and noise. At the same time, there will inevitably be power frequency interference and electromagnetic interference. This requires the suppression of the base drift circuit and the two filter circuit to filter the interference. The structure of the pulse oximeter circuit based on CMOS is shown in Fig.1.

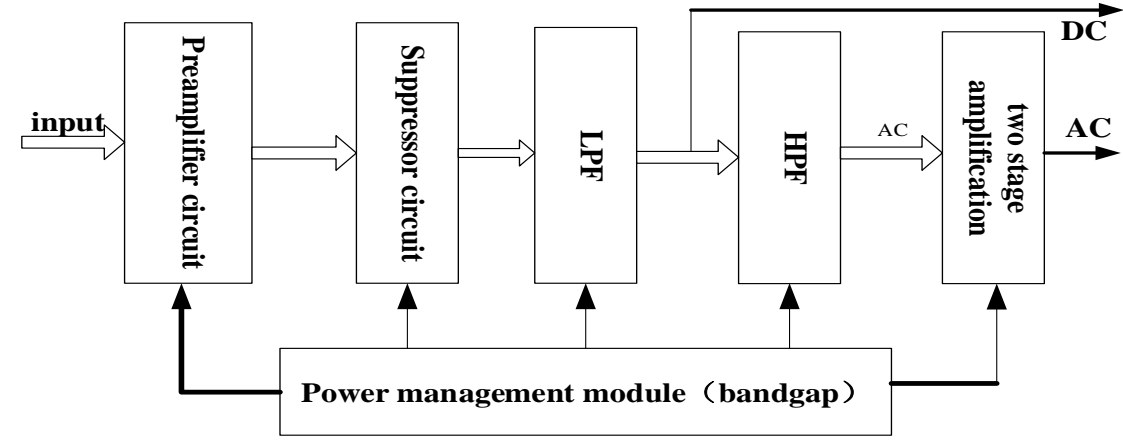

Fig.1 structure of the pulse oximeter circuit

\section{1 amplifier circuit}

The pulse signal collected by the pulse sensor is very weak, the signal amplitude is $20 \mathrm{mV} \sim 50 \mathrm{mV}$. In order to achieve the conversion voltage of ADC, the input signal of the circuit module should be increased by 50 80 times. In order to improve the anti-interference performance of the circuit (to avoid the distortion of the pulse signal caused by a large gain), the pulse oximeter measurement circuit is composed of preamplifier circuit and two-stage amplifier circuit. The preamplifier circuit is shown in Fig.2, the two-stage amplifier circuit is shown in Fig. 3. Among them, the preamplifier circuit uses differential amplifier. The differential amplifier circuit has a capability of strong common mode rejection $(82.5 \mathrm{~dB})$, which can suppress the temperature drift, voltage drift and effectively offset the random noise.

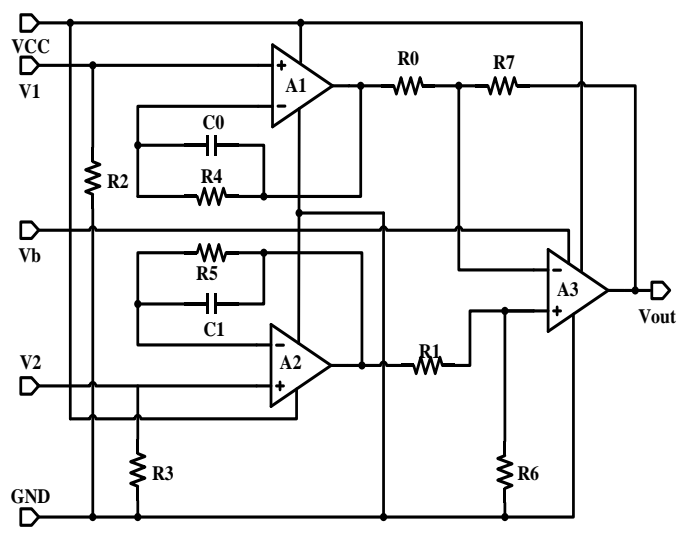

Fig.2 Preamplifier circuit

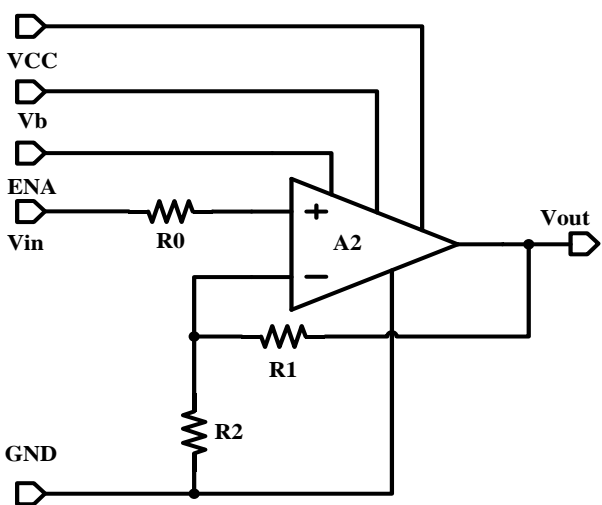

Fig.3 two-stage amplifier circuit 


\section{2 band pass filter}

After the signal is amplified, it needs to filter the interference. The band-pass filter circuit is designed to eliminate the interference and noise, including low-pass filter and high-pass filter. Low pass filter circuit uses two-order Butterworth low-pass filter circuit. This type of active circuit can not only remove the interference effectively, but also reduce the signal attenuation. At the same time, the signal can be amplified to some extent. The pulse signal after the low-pass filter circuit is composed of DC signal and AC signal. Among them, the DC signal is the volt level, while the AC signal is millivolt level. Therefore, it is necessary to amplify the AC signal individually. In this way, the AC and DC signals should be isolated, and then the AC signal can be amplified, some low-frequency interference can also be filtered out. Finally, the AC signal is fed into the two stage amplifier to obtain the value of $I_{\mathrm{AC}} 、 I_{\mathrm{DC}}$.

\section{System Design}

The pulse oximetry sensor system based on CMOS is composed of four modules: the reflective pulse oximetry module, data processing module, RFID transceiver module and the PC interface. In the process of data transmission, this system involves two kinds of communication modes including wired communication and wireless communication [4]. The communication between sensor and MCU, MCU and the tag, the reader and PC are all through the wired communication mode. The transmission of sensor data between RFID tag and RFID reader is accomplished by wireless communication. The data processing module will not only process the pulse and blood oxygen information [5,6] collected by the sensor, but also establish a connection with the RFID tag. At the same time, the pulse and blood oxygen data collected and processed are transferred to the register of the RFID tag. The data transmission between the sensor and the data processing module is through the serial port connection, and the data transmission between the data processing module and the RFID tag is communicated through the SPI interface.

RFID transceiver module includes the RFID active tags and the RFID reader. The RFID tag $[7,8]$ and the reader achieve the data transmission based on GEN IS0/IEC 18000-6C protocol. Based on the communication between the data processing modules, the whole communication process: MCU transmits the data in the register to the RFID tag through the SPI interface. The reader communicates with the tag in accordance with the radio frequency communication protocol and reads the data in the internal register of the RFID tag. Finally the RFID reader read the data in the RFID tag through wireless communication [9], and display the data on PC interface. The overall design structure diagram of pulse oximetry system is shown in Fig.4.

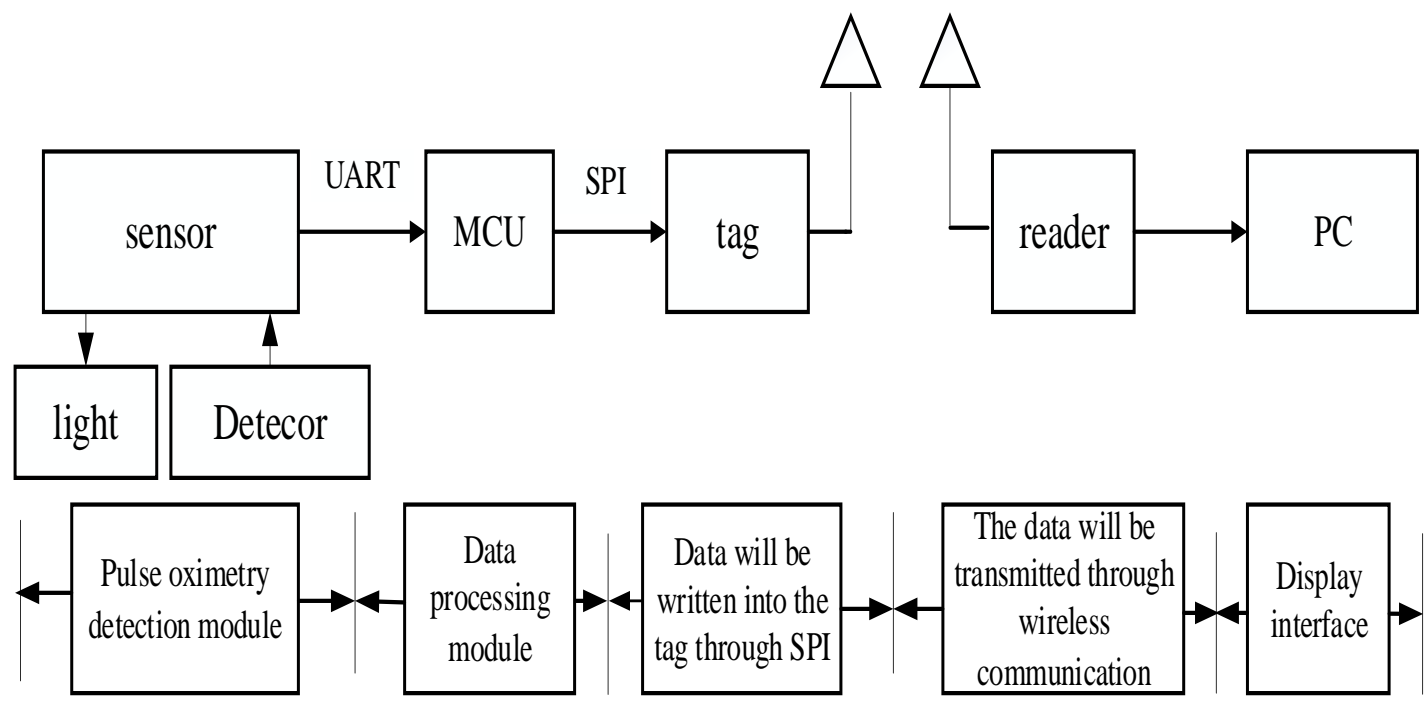

Fig.4 the structure diagram of pulse oximetry system

\section{Process of The Data Transmission}

After the reader and tag are connected, the pulse oximeter system will be tested. The pulse oximeter sensor is connected with the host computer through the USB interface, which can directly display the red light and infrared light PPG signal measured by the sensor. According to the principle of dual wavelength detection, the PPG signal can be transformed into digital signal, which can be used for further transmission of the data. The PPG signal waveform of the sensor is shown in Fig.5. 


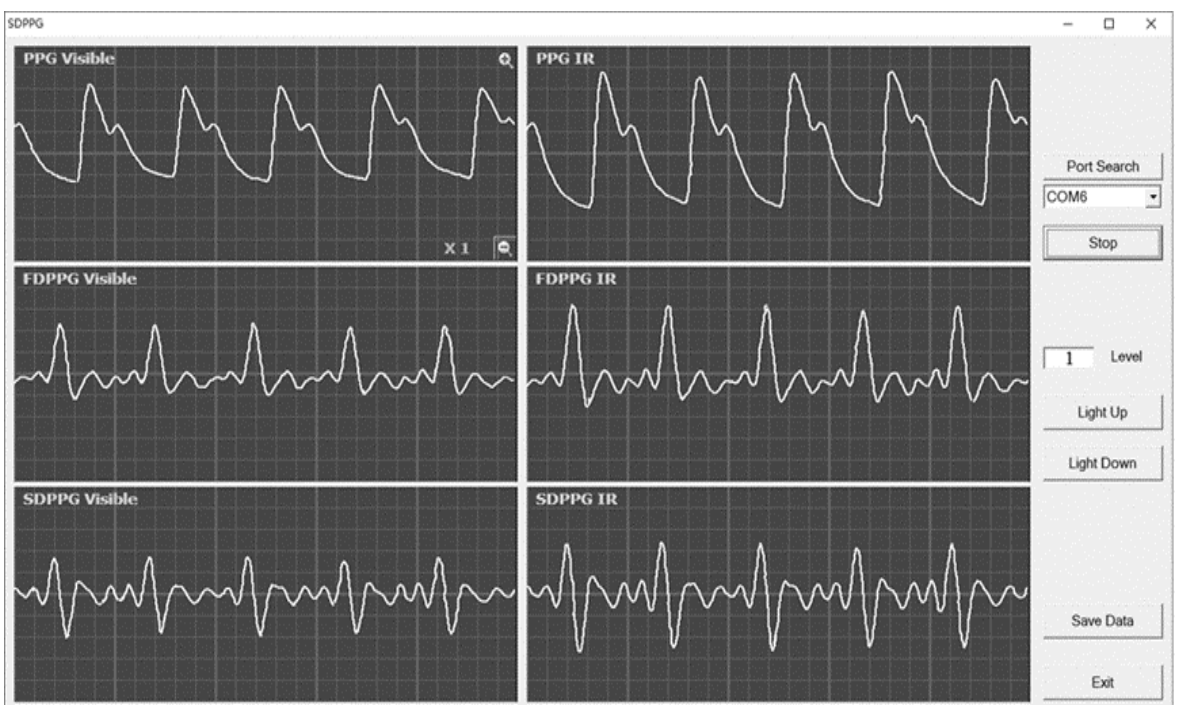

Fig. 5 waveform of PPG signal collected by sensor

In the pulse oximetry system, the pulse oximeter is connected with the data processing module through the UART interface. The data processing module is connected with the RFID tag through the SPI interface. Then the data in the register of data processing module is transferred to the internal register of the tag. The RFID reader and RFID tags constitute the RFID transceiver module, it works in the $860-960 \mathrm{MHz}$ frequency range. The data transmission is based on the EPC C1 GEN2/ISO 18000-6C protocol. The reader interface is shown in Fig.6, and the reader starts working after clicking on the connection button. Click on the label and open a single label cycle recognition mode, the reader will identify the tag within the working range. The data window displays the label number, storage area and storage address of the tag identified by the reader.

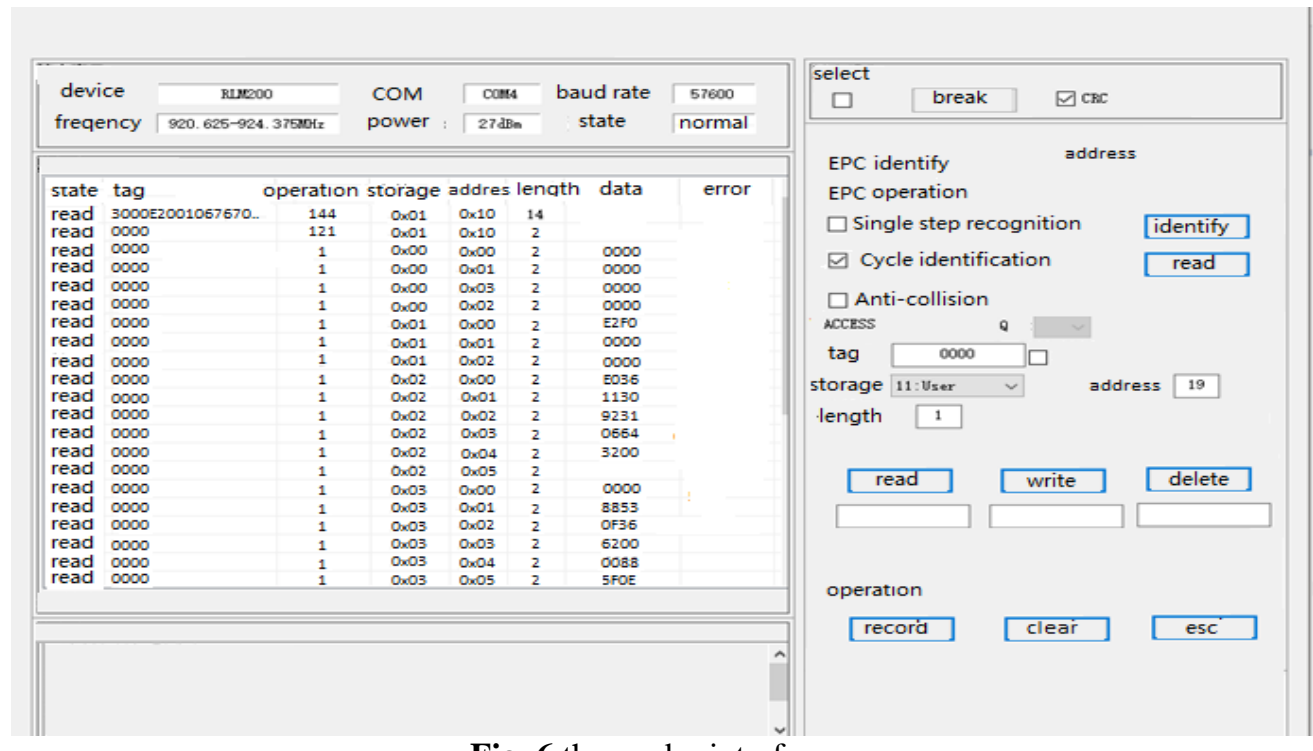

Fig. 6 the reader interface

The data read by the reader is shown in Fig.6, the contents of the data window in the graph are the tag information identified by the reader and the data in each storage area. The reader is connected with the host computer through the USB interface, which can display the value and waveform of the pulse and oxygen saturation in the computer interface. The pulse oximeter information displayed on the computer interface is shown in Fig.7. 


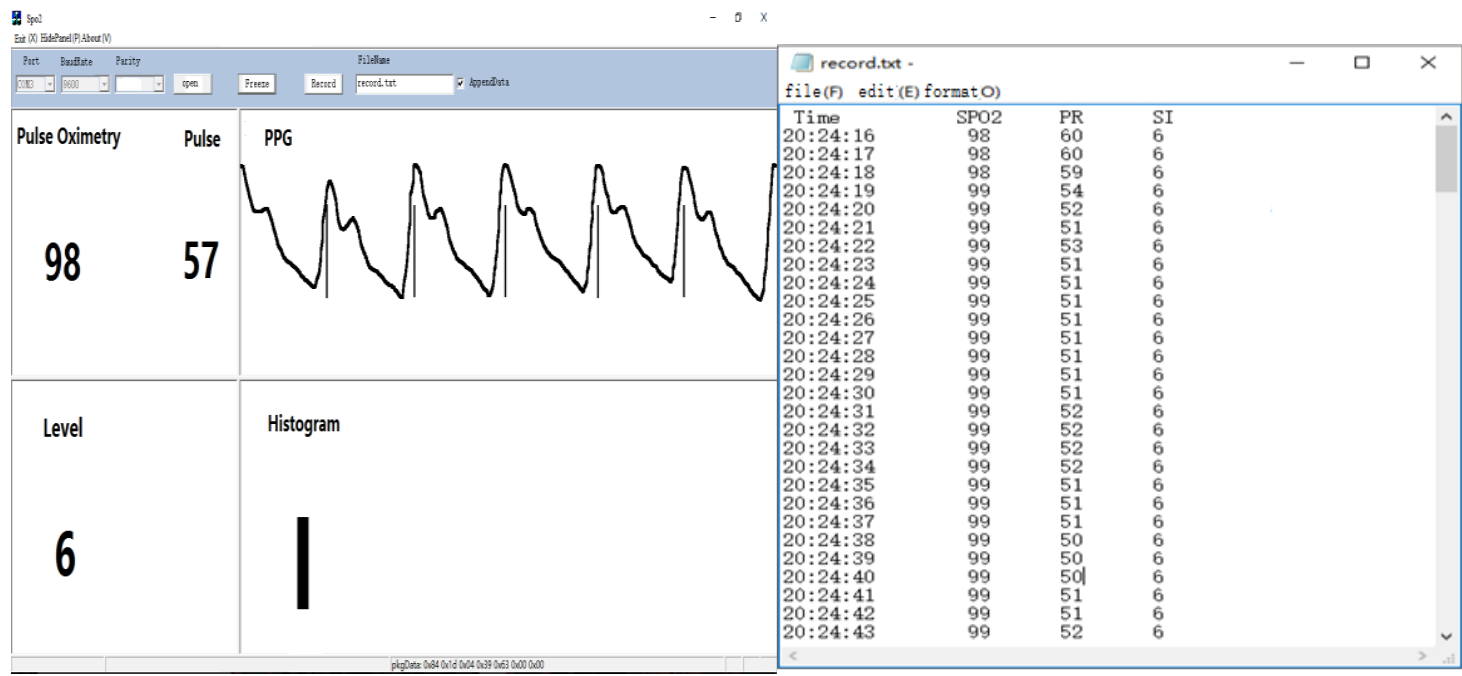

Fig. 7 the information displayed in the computer interface

Fig. 8 Pulse oximetry data

Click on the record, the pulse and oxygen saturation data received by the host computer can be saved in the form of text. The real time data detected by the pulse oximeter system is shown in Fig.8. Users can check their information on a regular basis. By analyzing and processing the real time data of pulse and blood oxygen saturation, we can know the health status and the development trend. It can play a good role in monitoring the health of the user.

\section{Experimental Validation}

After the system hardware and software debugging, the finger probe BSJ09001C of Shanghai Berry Company was chosen in order to further verify the feasibility and accuracy of the system. Then the measured data are compared with the data obtained by the system. The measurement range of oxygen saturation is $35 \%-100 \%$, and the measurement error is $\pm 1 \%$. The pulse rate measurement range is $25-250 \mathrm{bpm}$, the measurement error is $\pm 2 \% .50$ people of different ages were selected for the test, and each person had no strenuous exercise before the test. The subjects were given at least five minutes before the signal extraction. During the measurement process, the room needs to be quiet, and the results are recorded after the measurement data is stable. Then there will be a comparison and analysis of the data between the system and the oximeter. The results of oxygen saturation and pulse rate are shown in Fig.9 (a), (b). Among them, series 1 represents the data measured by the oxygen sensor, and series 2 represents the data measured from the system.

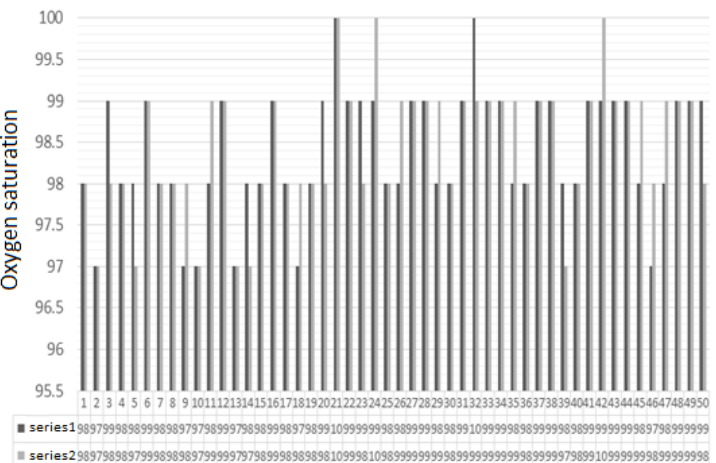

(a)

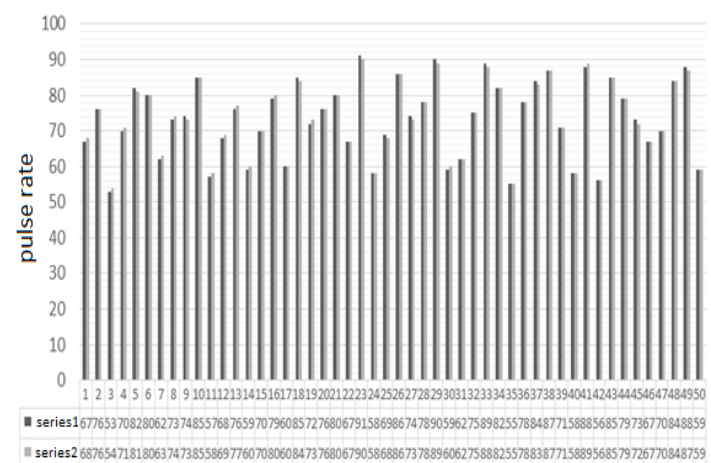

(b)

Fig.9 Results of blood oxygen saturation and pulse rate.

The error analysis of the results of blood oxygen saturation and pulse measurement is shown in Fig.10, in which the series 1 and 2 represent the percentage error of pulse rate and oxygen saturation respectively. It can be concluded that the maximum error is $\pm 1 \%$, and the maximum error of pulse rate is $\pm 1.9 \%$.The experimental objects are selected scientifically, and the test results are representative. Although there are individual differences in the oxygen saturation and pulse rate of the test object, the transmission stability of the reflective pulse oximetry system can be guaranteed. The accuracy of oxygen saturation and pulse rate is: the error range of oxygen saturation is $\pm 1 \%$, and the error range of pulse rate is $\pm 2 \%$.It can be seen that the reflective pulse oximetry 
sensing system has good stability and accuracy. The experimental results show that the accuracy and reliability of the system meet the design requirements, and it is proved that the system can be used to monitor the pulse and blood oxygen saturation.

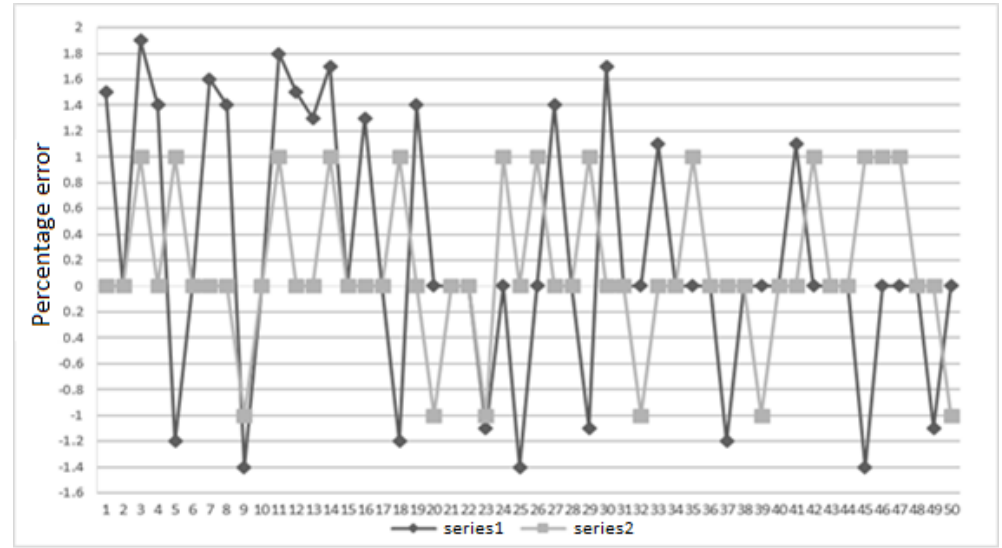

Fig.10 the results of error analysis of the system

\section{Conclusion}

The pulse oximetry circuit and its sensing system is designed based on the CMOS technology. The principle of pulse oximetry circuit is analyzed, and the corresponding circuit is designed to realize the acquisition and amplification of pulse oximeter signal. It can realize the function of real-time monitoring of pulse oximetry in the middle and far distance with the combination of microprocessor and wireless sensor technology. The experimental results show the feasibility of the proposed scheme, and the system has the advantages of stable operation, high precision and low power consumption. With the development of science and technology and the continuous improvement of the medical level, the characteristics of miniaturization and wireless monitoring have a good prospect in the field of wearable medical research.

\section{Acknowledgments}

This research is funded by Tianjin Research Program of Application Foundation and Advanced Technology (No. 15JCYBJC16300), and Tianjin science and Technology Commissioner Project (No. 16JCTPJC45500).

\section{References}

[1] Y. Mendelson, Noninvasive pulse oximetry utilizing skin reflectance photo plethysmography. IEEE Transactions on Biomedical Engineering, 35 (10), 1988, 798-805

[2] V. Kirk, S. Bohn, W. Flemons, and J. Rammers, Comparison of home oximetry monitoring with laboratory polysomnography in children. Amer. College Chest Phys, 2003, 1702-1708

[3] S. Ley, D. Laqua, P. Husar, Simulation of Photon Propagation in Multi-layered Tissue for Non-invasive Fetal Pulse Oximetry, The 15th International Conference on Biomedical Engineering, 2013, 356-359

[4] S. Warren, J Lebak, J Yao, at al, Interoperability and security in wireless body area network infrastructures [C], Conf Proc IEEE Eng Med Biol Soc, 2005, $837-840$

[5] T. Polk, W. Walker, A Hande, Wireless telemetry for oxygen saturation measurements [A] .IEEE Biomedical Circuits and Systems (BiOCAS) Conference Proceedings [C],London , UK ,November , 2006.

[6] S. Arms, C. Townsend, C. Churchill, ,Power management for energy h harvesting wireless sensors [A] .SPIE International Symposium on Smart Structures and Smart Materials Proceedings [C], 2005.

[7] S.LEE, S.JOO, C.LEE, An enhanced dynamic framed slotted ALOHA algorithm for RFID tag[C].Proceedings of The Second Annual International Conference on Mobile and Ubiquitous Systems: Networking and Services, Washington, DC: IEEE Computer Society, 2005,166-172

[8] Dirk Hihnel, Wolfram Burgard, Dieter Fox, et al, Mapping and Localization with RFID Technology[C].IEEE international Conference on robotics on \& automation, 2004, 1015-1020

[9] W. Choi and D I Oh. R-LIM, an Affordable Library Search System Based on RFID[C]. International Conference on Hybrid Information Technology, Cheju Island, 2006, 103-108 